\title{
Dyslipidemia Modified the Association Between Serum Uric Acid and First Hemorrhagic Stroke Among Chinese Population : A Community Based Nested Case-control Study
}

\author{
Hui Liu \\ Department of Cardiovascular Medicine冈the Second Affiliated Hospital of Nanchang University $₫$ Nanchang of Jiangxi凶China \\ Chonglei Bi \\ People's Hospital of Rongcheng,Shandong ,China \\ Tengfei Lin \\ Beijing Advanced Innovation Center for Food Nutrition and Human Health,College of Food Science and Nutritional Engineering,China Agricultural \\ University,Beijing,China \\ Lishun Liu \\ Beijing Advanced Innovation Center for Food Nutrition and Human Health,College of Food Science and Nutritional Engineering,China Agricultural \\ University,Beijing, China \\ Chengzhang Liu \\ Shenzhen Evergreen Medical Institute,Shenzhen,China \\ Ping Wang \\ School of Public Health (Shenzhen),Sun Yat-Sen University,Guangzhou,China \\ Yun Song \\ Beijing Climate Center for Food Nutrition and Human Health,College of Food Science and Nutritional Engineering,China Agricultural University, Beijing,China. \\ School of Public Health (ShenZhen), Sun Yat-Sen University,Guangzhou,China)) \\ Binyan Wang \\ Shenzhen Evergreen Mediccal Institute,Shenzhen,China. Institute of Biomedicine, Anhui Medical University,Hefei,China \\ Chongqian Fang \\ Peolpe's Hospital of Rongcheng,Shandong,China \\ Hai Ma \\ Health and Family Planning Commission,Rongcheng,Shandong,China \\ Xiao Huang \\ Department of Cardiovascular Medicine, the Second Affiliated Hospital of Nanchang University,Nanchang of Jiangxi, China \\ Xiping Xu \\ Beijing Advanced Innovation Center for Food Nutrition and Human Health,College of Food Science and Nutritional Engineering,China Agricultural \\ University,Beijing,China. National Clinical Research Study Center for Kidney Disease. the State Key Laboratory for Organ Failure Research. \\ Huiyuan Guo \\ Beijing Advanced Innovation Center for Food Nutrition and Human Health,College of Food Science and Nutritional Engineering,China Agricultural \\ University,Beijing,China

\section{Xiaobin Wang} \\ Department of Population,Family and Reproductive Health,Johns Hopkins University Bloomberg School of Public Health,Baltimore,MD,USA.

\section{Huihui Bao} \\ Center for Prevention and Treatment of Cardiovascular Diseases,the Second Affiliated Hospital of Nanchang University, Nanchang of Jiangxi,China \\ Xiaoshu Cheng ( $\nabla$ xiaoshumenfan126@163.com) \\ Center for Prevention and Treatment of Cardiovascular Diseases, the Second Affiliated Hospital of Nanchang University, Nanchang of Jiangxi, China. \\ https://orcid.org/0000-0001-7445-1988
}

\section{Research}

Keywords: dyslipidemia, serum uric acid, hemorrhagic stroke, nested case-control study

Posted Date: December 11th, 2020

DOI: https://doi.org/10.21203/rs.3.rs-124307/v1

License: (1) This work is licensed under a Creative Commons Attribution 4.0 International License. Read Full License 


\section{Abstract}

Background: Previous studies have shown that serum uric acid (SUA) and dyslipidemia are risk factors for stroke. However, it is not clear that whether dyslipidemia could modify the association between SUA and hemorrhagic stroke.

Methods: We conducted a nested case-control study from "H-type Hypertension and Stroke Prevention and Control Project (HSPCP)" in China. A total of 355 first hemorrhagic stroke cases and 355 controls matched for age ( \pm 1 years), sex and study site were included in the final data analysis. Logistic regression analysis were performed to assess the association between SUA and first hemorrhagic stroke.

Results: In matched analysis, the risk of hemorrhagic stroke was associated with the increased SUA levels (OR:1.13, 95\% Cl:0.98 to 1.30). However, multiple regression analyses showed that per $1 \mathrm{mg} / \mathrm{dL}$ in SUA was associated with a 16\% decreased risk of hemorrhagic stroke among participants without dyslipidemia and associated with a $28 \%$ increased risk of hemorrhagic stroke among dyslipidemia participants [without dyslipidemia: odds ratio (OR) $=0.84$, $95 \% \mathrm{Cl} 0.65$ to 1.09 , with dyslipidemia: $\mathrm{OR}=1.28,95 \% \mathrm{Cl} 1.10$ to $\left.1.48 ; P_{\text {Interaction }} \otimes 0.001\right]$. The results of the subgroup analysis were consistent with the main results.

Conclusions: Elevated SUA was associated with increased risk of hemorrhagic stroke especially among participants with dyslipidemia. These findings suggest that we need to focus on SUA levels especially among participants with dyslipidemia.

\section{Introduction}

Stroke is the main cause of death in China and the second leading cause of death in the world ${ }^{[1]}$. In 2016 , the global lifetime risk of stroke from the age of 25 years onward was approximately $25 \%$ among both men and women ${ }^{[2]}$. A Large number of epidemiological studies indicate that $70 \%$ of strokes are caused by hypertension, which is the most common controllable factor for stroke ${ }^{[3]}$. Although the control rate of hypertension has increased, the incidence of stroke remains high. In addition to controlling traditional risk factors, therefore, it is necessary to identify novel, modifiable markers to inform the risk of stroke, with the goal to achieve primary prevention better.

Serum uric acid (SUA) is a product of human purine metabolism. A large number of studies have shown that SUA was related to hypertension ${ }^{[4]}$, metabolic syndrome ${ }^{[4]}$, kidney disease ${ }^{[5]}$, heart failure ${ }^{[5]}$, stroke ${ }^{[5]}$ and other diseases ${ }^{[8,9]}$, but it was uncertain whether SUA was an independent risk factor for stroke. Some studies found a positive correlation between SUA and stroke ${ }^{[10,11]}$, while others not ${ }^{[12,13]}$. It is worth mentioning that most studies focused on the association between SUA and ischemic stroke, and there are few studies on SUA and hemorrhagic stroke. Although hemorrhagic stroke is not as common as ischemic stroke, it has a higher risk of death than ischemic stroke ${ }^{[14]}$. To our knowledge, dyslipidemia is also a risk factor for hemorrhagic stroke ${ }^{[15-17]}$ and it was reported that serum uric acid was positively correlated with cholesterol, triglycerides and low density lipoprotein, while negatively correlated with low density lipoprotein ${ }^{[18,19]}$. However, it is not clear that whether dyslipidemia could modify the association between SUA and hemorrhagic stroke. Hence, our study aimed to investigate the possible relationships between serum uric acid and hemorrhagic stroke in people with or without dyslipidemia.

\section{Methods}

Study design and populations

Our study data came from the "H-type Hypertension and Stroke Prevention and Control Project (HSPCP)" which was a non-intervention, observational, realworld registry study. It was planed to assess the incidence and control of hypertension in China and the related factors affecting its prognosis, and to build the risk prediction model of cardio-cerebral and renal vascular diseases finally. Men or women aged $\geq 18$ years with a sitting systolic blood pressure (SBP) $\geq 140$ $\mathrm{mmHg}$ and/or a sitting diastolic blood pressure $\mathrm{BP}(\mathrm{DBP}) \geq 90 \mathrm{~mm} \mathrm{Hg}$, or with suspected hypertension at the screening visit were eligible. If Participants were unable to show informed consent due to psychological or neurological impairment or were unable to follow up according to the study protocol, they would be excluded. Screening and recruitment and a 5-year observation follow-up period were involved. Blood pressure, heart rate, usage of drugs, adverse events and study outcome events were recorded during the follow-up of each year as planned. The primary outcome was composite of cardiovascular events consisting of nonfatal stroke, myocardial infarction, and vascular death and all-cause death.

All stroke endpoints were from the local the Center for Disease Control and Prevention. Stroke and non-stroke were matched according to $1: 1$ by age \pm 1 year, same gender, and same town. The initial sample consisted of 3771 incident cases and 3771 matched controls. Next, we excluded participants with missing serum calcium value $(n=450)$. Based on the inclusion and exclusion criteria, 3546 stroke cases and 3546 matched controls with complete were selected for final data analysis $₫$ Supplementary Figure $1 \rrbracket$. In the current study, we explored the relationship between serum uric acid and hemorrhagic stroke. Therefore, 355 patients with hemorrhagic stroke and their matched were eventually analyzed in the exclusion of other types of stroke and people taking lipid-lowering

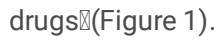

\section{Outcome}

The primary outcome of this study was a first nonfatal or fatal hemorrhagic stroke. Information on incidence of stroke for all participants was obtained via the Center for Disease Control and Prevention of Rongcheng and Lianyungang city and checked against the national health insurance system with electronic linkage to all hospitalizations, or confirmed through active follow-up. International Classification of Diseases, 10th Revision (ICD-10) was used to coded all diseases. The primary outcome (first nonfatal or fatal stroke) included first hemorrhagic stroke (160-162). In this study, patients with subarachnoid hemorrhagic (160) were not in the analysis. 
Local authorities at medical institutions were required to report all new stroke cases to the local centers for Disease Control and prevention. A report card attached information about demographics, diagnostic basis and date of stroke was required to be submitted on the 28th of each month. Quality control of case was checked by experienced officials. Furthermore, the local CDC was also in charge of deleting repeated cases and discovering logistical errors and missed cases. Moreover, $5 \%$ of all uploaded cases are randomly chosen for further confirmation on the phone or door-to-door interviews.

\section{Covariates}

At this baseline assessment, participants reported on their lifestyle, medical conditions and demographic information; medical history, health status, and medication intake were queried by trained health professionals. The anthropometric examinations included weight, height. Body mass index (BMI) was calculated as the body weight in kilograms divided by the square of the height in meters $\left(\mathrm{kg} / \mathrm{m}^{2}\right)$. Continuous variables included age (years), BMI ( $\left.\mathrm{kg} / \mathrm{m}^{2}\right)$, blood pressure (BP, mmHg) and biochemical data [fasting blood glucose (FBG, mmol/L), total cholesterol (TC, mmol/L), triglycerides (TG, mmol/L), high density lipoprotein cholesterol (HDL-C, mmol/L), homocysteine (Hcy, $\mu \mathrm{mol} / \mathrm{L})$, serum uric acid (SUA, mg/dL) and estimated glomerular filtration rate (eGFR, $\left.\mathrm{mL} \cdot \mathrm{min}^{-1} \cdot 1.73 \mathrm{~m}^{-2}\right)$ ]. Categorical variables consisted of sex (male, female), smoking (no, yes), drinking (no, yes). EGFR was estimated using the newly developed CKD-EPI equation ${ }^{[20]}$.

\section{Definition of dyslipidemia}

According to $\square$ Guidelines for Prevention and Treatment of Chinese Adults with Dyslipidemia in 2016 ${ }^{[21]}$, abnormal levels of any of total cholesterol, triglycerides, low-density lipoprotein, and high-density lipoprotein can be considered as dyslipidemia:1) total cholesterol is greater than or equal to $5.2 \mathrm{mmol} / \mathrm{L}$, 2) low-density lipoprotein is greater than or equal to $3.4 \mathrm{mmol} / \mathrm{L}$, triglyceride is equal to or greater than $1.7 \mathrm{mmol} / \mathrm{L}$, high density lipoprotein is less than 1.0 $\mathrm{mmol} / \mathrm{L}$.

\section{Statistical analysis}

Data are presented as mean \pm SD for continuous variables and as frequency (\%) for categorical variables. Differences in baseline characteristics between cases and controls were compared using generalized paired t-tests for continuous variables and chi-square tests for categorical variables. Taking SUA and dyslipidemia as independent variables respectively and first hemorrhagic stroke as an dependent variable, logistic regression analysis was performed to assess the odds ratio $(\mathrm{OR})$ and $95 \%$ confidence interval $(\mathrm{Cl})$. At the same time, the association between SUA and first hemorrhagic stroke was examined stratified by without or with dyslipidemia. Potential confounders included in the logistic regression models, other than the matching criteria, were BMI, smoking, drinking, SBP, DBP, FBG, eGFR and anti-hypertensive drugs.

All data analysis used R software version 4.0.3 (https://www.R-project.org) and Empower (R) version 3.0 (www.empowerstats.com). A two-sided $P<0.05$ was defined as significant differences.

\section{Results}

\section{Baseline Characteristics of Study Participants.}

As shown in figure 1, a total of 355 hemorrhagic stroke and their matched individuals were enrolled in this study. Baseline characteristics of study participants were presented in Table 1. In the general population, patients in cases group were tend to have higher systolic blood pressure (SBP), diastolic blood pressure (DBP), serum uric acid (SUA), fasting blood glucose (FBG) and total cholesterol (TC) compared with controls group. When the baseline characteristics were presented by with or without dyslipidemia, the difference of SUA between cases and controls was observed only in those with dyslipidemia (case: $6.0 \pm 1.7$ $\mathrm{mg} / \mathrm{dL}$,control: $5.5 \pm 1.4 \mathrm{mg} / \mathrm{L}, P<0.001)$.

\section{Association between SUA and hemorrhagic stroke.}

Table 2 showed the association of first stroke with SUA using conditional logistic regression analyses. After adjustment for confounding factors, for per SD increment in SUA, the risk of first stroke increased by $13 \%(\mathrm{OR}=1.13,95 \% \mathrm{Cl}$ : $0.98-1.30)$. We also converted SUA from a continuous variable to a categorical variable (tertiles). Compared to SUA $<4.8 \mathrm{mg} / \mathrm{dL}$, the adjusted OR of first stroke for participants in T3 ( $\geq 6.1 \mathrm{mg} / \mathrm{dL}$ ) was 1.44 ( $95 \% \mathrm{Cl}$ : $0.88,2.35$ ). $P$ for trend in the adjusted models was not significant. For people without dyslipidemia, there was no significant association between SUA and hemorrhagic stroke, whether SUA was analyzed as a continuous or categorical variable. For people with dyslipidemia, for per SD incremengt in SUA, the risk of hemorrhagic stroke increased by $28 \%(\mathrm{OR}=1.28,95 \% \mathrm{Cl}, 1.10-1.48)$. After SUA tertiles, using low levels of SUA as a reference, we found that the higher the SUA level, the greater the risk of stroke, especially when the SUA was higher than $6.1 \mathrm{mg} / \mathrm{dl}$, the risk of stroke was increased by $84 \%(\mathrm{OR}=1.84,95 \% \mathrm{Cl}, 1.10-3.09)$. $P$ for trend in the all models was significant and consistent with the $P$ value when SUA was used as a continuous variable, suggesting the linear association between SUA and first stroke when patients accompanied with dyslipidemia. What's more, $P$ value for interaction of with or without dyslipidemia on between SUA (continuous) and hemorrhagic stroke $₫ 0.001$. The results of the relationship between dyslipidemia and hemorrhagic stroke was presented in Table S1. Taking people without dyslipidemia as a reference, people with dyslipidemia had a higher risk of hemorrhagic stroke $₫ \mathrm{OR}=1.19,95 \% \mathrm{Cl}(0.83-2.04)$. Although supplementary table 1 revealed the relationship between dyslipidemia and hemorrhagic stroke in our study, it was not statistically significant. Further analyses using restricted cubic spline confirmed the dose-relationship between SUA and hemorrhagic stroke presented by with or without dyslipidemia (Figure 2). It could be seen that the SUA in the dyslipidemia group was positively correlated with hemorrhagic stroke. Although the curve of SUA and hemorrhagic stroke was negatively correlated in patients without dyslipidemia, it had no statistical significance and might be affected by sample size.

We also explored the detailed impact of lipid components on the association between SUA and hemorrhagic stroke. As shown in supplementary table 2, of the four components included in the analysis, TC,TG, LDL-C had a modifying effect on the relationship between SUA and hemorrhagic stroke except for the HDL-C 


\section{Subgroup analysis}

We further explored the role of other covariables on the association between SUA and hemorrhagic stroke. As shown in Figure 3, the subgroup analysis

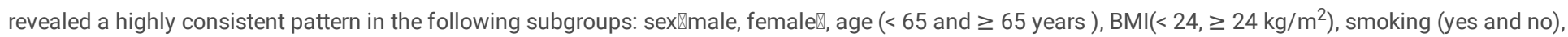
drinking (yes and no), eGFR $\left(<90, \geq 90 \mathrm{ml} / \mathrm{min} / 1.73 \mathrm{~m}^{2}\right.$ ) and SBP $(<140, \geq 140 \mathrm{mmHg}$ ) (all $P$ for interaction $>0.05$ ).

\section{Discussion}

This nested case-control study showed that dyslipidemia could modify the association between SUA and hemorrhagic stroke. The results showed a positive correlation between SUA and hemorrhagic in the patients with dyslipidemia, but there was no statistically significant difference in patients without dyslipidemia.

Previous studies have reported the relationship between SUA and hemorrhagic stroke, but the results were controversial. The Rotterdam study ${ }^{\text {[22] }}$ which was followed up for 8.4 years found that high serum uric acid levels were associated with hemorrhagic stroke, but it was not statistically significant (HR: 1.68 , 95\% $\mathrm{Cl}: 0.68$ to 4.15). At the same time, a U-shaped relationship was found between SUA and hemorrhagic stroke in a study, but it was also not statistically significant ${ }^{[23]}$. The possible reason might be insufficient sample size. Moreover, a prospective study ${ }^{[23]}$ based on data of China Stroke Primary Prevention Trial recruited 20,577 participants with hypertension who had no history of stroke and were followed up for 4.5 years. It found that a statistically significantly decreased risk of hemorrhagic stroke for the second SUA quartile (Q2) compared to the first quartile (Q1) (HR 0.56, 95\%Cl: 0.32-0.97, P = 0.037). These contradictory results might be due to different trial designs, populations in different regions, and adjustment factors.

A positive correlation between SUA and hemorrhagic in the patients with dyslipidemia was found in our prestent study, but not in those without dyslipidemia. This might suggested that different blood lipid levels had an effect on SUA. According to reports, the use of Finofibrate (a drug to lower triglyceride) could reduce the concentration of SUA by increasing the urinary excretion ${ }^{[24]}$. Atorvastatin had also been shown to lower the level of SUA ${ }^{[25]}$. These results might suggest that elevated cholesterol or triglyceride without timely medication would lead to accumulation of SUA, and resulting in eventually stroke.

To our knowledge, SUA was related to hypertension which was a common cause of cerebral hemorrhage. A study proved that TG could strengthen the effect of SUA on blood pressure ${ }^{[25]}$. In addition, the role of SUA in humans has been previously studied. It has been linked to increased levels of inflammatory markers such as interleukin-6, interleukin-18, c-reactive protein and tumor necrosis factor-alpha ${ }^{[25]}$, which could damage vascular endothelial function through oxidative stress. What's more, SUA could promote lipids oxidation and further damage vascular endothelium ${ }^{[28]}$. Damaged endothelial cells are more likely to develop into small aneurysms ${ }^{[29]}$. These might also explain the reason that people with abnormal lipids levels had a more significant correlation between SUA and hemorrhagic stroke.

Current study found that dyslipidemia modified the relationship between SUA and hemorrhagic stroke. Of course, several limitations should not be ignored. First, SUA concentrations and blood lipids level of all participants were only assessed at baseline and and their dynamic changes during follow-up were not taken into account. Second, we did not have detailed information of food and drugs intake which affected SUA levels at baseline and during the course of follow-up. Third, this was a nested case-control study with a relatively small samples sizes among a community-based population at coastal areas of China, further larger-scale cohort studies and randomized trials were need to confirm the findings and assess the applicability of the results for other populations. Finally, as far as current research was concerned, our findings required more accurate pathogenesis to explain.

\section{Conclusion}

In sunmary, elevated SUA was associated with increased risk of hemorrhagic stroke especially among participants with dyslipidemia. These findings suggest that we need to focus on SUA levels especially among participants with dyslipidemia.

\section{Declarations}

\section{Ethics approval and consent to participate}

The study was approved by the Ethics Committee of the Institute of Biomedicine, Anhui Medical University, Hefei, China. All participants signed an approved written consent after it was explained to them.

\section{Availability of data and materials}

Data sharing is not applicable to this article as no datasets were generated or analysed during the current study.

\section{Author Contributions}

Conceptualization, HL, XSC, HHB, XPX, XBW and XH.; methodology, CLB, TFL, LSL, PW, YS, CQF and BYW; software, HL, HM and XH; formal analysis, HL, HYG and HHB; investigation, CLB, LSL, HM, TFL, PW, YS, BYW, HHB, XPX and XBW; resources, XPX, XH and; data curation, CZL, XBW, XPX and XSC; writing-Original draft preparation, HL; writing-Review and editing, XBW, HHB and XSC; visualization, XPX; supervision, CZL, LSL, HHB and XPX; project administration, LSL, BYW and XPX; funding acquisition, $\mathrm{XPX}$ and $\mathrm{XH}$. All authors have read and agreed to the published version of the manuscript. 


\section{Acknowledgements}

We acknowledge the contribution the all staff who participated in this study as well as the study participants who shared their time with us

\section{Conflict of interest disclosures}

The authors declare that they have no competing interests

\section{Funding}

Dr. Xiping Xu reports grants from the National Key Research and Development Program [2016YFE0205400, 2018ZX09739, 2018ZX09301034003), the Science and Technology Planning Project of Guangzhou, China (201707020010), the Science Technology and Innovation Committee of Shenzhen [JSGG20170412155639040, GJHS20170314114526143, JSGG20180703155802047), the Economic Trade and Information Commission of Shenzhen Municipality [20170505161556110, 20170505160926390]. Dr. Xiao Huang reports grants from the National Natural Science Foundation of China [81960074, 81500233], Jiangxi Outstanding Person Foundation [20192BCBL23024], Major projects of the Science and Technology Department, Jiangxi [20171BAB205008]. No other disclosures were reported.

\section{References}

[1] Lozano R, Naghavi M, Foreman K, Lim S, Shibuya K, Aboyans V, et al. Global and regional mortality from 235 causes of death for 20 age groups in 1990 and 2010: a systematic analysis for the Global Burden of Disease Study 2010. Lancet, 2012,380(9859):2095-2128.

[2] Feigin VL, Nguyen G, Cercy K, Johnson CO, Alam T, Parmar PG, et al. Global, Regional, and Country-Specific Lifetime Risks of Stroke, 1990 and 2016. N Engl J Med, 2018,379(25):2429-2437.

[3] Whitworth JA. 2003 World Health Organization (WHO)/International Society of Hypertension (ISH) statement on management of hypertension. J Hypertens, 2003,21(11):1983-1992.

[4] Ford ES, Li C, Cook S, Choi HK. Serum concentrations of uric acid and the metabolic syndrome among US children and adolescents. Circulation, 2007,115(19):2526-2532.

[5] Siu YP, Leung KT, Tong MK, Kwan TH. Use of allopurinol in slowing the progression of renal disease through its ability to lower serum uric acid level. Am $J$ Kidney Dis, 2006,47(1):51-59.

[6] Mantovani A, Targher G, Temporelli PL, Lucci D, Gonzini L, Nicolosi GL, et al. Prognostic impact of elevated serum uric acid levels on long-term outcomes in patients with chronic heart failure: A post-hoc analysis of the GISSI-HF (Gruppo Italiano per lo Studio della Sopravvivenza nella Insufficienza Cardiaca-Heart Failure) trial. Metabolism, 2018,83:205-215.

[7] Zhang S, Liu L, Huang Y Q, et al. The association between serum uric acid levels and ischemic stroke in essential hypertension patients[J]. Postgrad Med, 2020:1-8.

[8] Schretlen DJ, Inscore AB, Vannorsdall TD, Kraut M, Pearlson GD, Gordon B, et al. Serum uric acid and brain ischemia in normal elderly adults.Neurology, 2007,69(14):1418-1423.

[9] Roberts JM, Bodnar LM, Lain KY, Hubel CA, Markovic N, Ness RB, et al. Uric acid is as important as proteinuria in identifying fetal risk in women with gestational hypertension. Hypertension, 2005,46(6):1263-1269.

[10] Li J, Muraki I, Imano H, Cui R, Yamagishi K, Umesawa M, et al. Serum uric acid and risk of stroke and its types: the Circulatory Risk in Communities Study (CIRCS). Hypertens Res, 2020,43(4):313-321.

[11] Strasak A, Ruttmann E, Brant L, Kelleher, C, Klenk J, Concin H, et al. Serum uric acid and risk of cardiovascular mortality: a prospective long-term study of 83,683 Austrian men. Clin Chem, 2008,54(2):273-284.

[12] Zhang W, Iso H, Murakami Y, Miura K, Nagai M, Sugiyama D, et al. Serum Uric Acid and Mortality Form Cardiovascular Disease: EPOCH-JAPAN Study. J Atheroscler Thromb, 2016,23(6):692-703.

[13] Shi X, Yang J, Wang L, Zhao M, Zhang C, He M, et al. Prospective study of serum uric acid levels and stroke in a Chinese hypertensive cohort. Clin Exp Hypertens, 2017,39(6):527-531.

[14] Lloyd-Jones D, Adams R, Carnethon M, De Simone G, Ferguson TB, Flegal K, et al. Heart disease and stroke statistics-2009 update: a report from the American Heart Association Statistics Committee and Stroke Statistics Subcommittee. Circulation, 2009,119(3):480-486.

[15] Wang X, Li S, Bai Y, Fan X, Sun K, Wang J, et al. Inverse association of plasma level of high-density lipoprotein cholesterol with intracerebral hemorrhage. J Lipid Res, 2011,52(9):1747-1754.

[16] Ma C, Gurol ME, Huang Z, Lichtenstein AH, Wang X, Wang Y, et al. Low-density lipoprotein cholesterol and risk of intracerebral hemorrhage: A prospective study. Neurology, 2019,93(5):e445-e457. 
[17] Krel M, Miulli DE, Jung H, Wiginton JG Th, Brazdzionis J, Wacker MR, et al. Minimization of Intraparenchymal Hemorrhagic Stroke Size by Optimization of Serum Lipids. Cureus, 2019,11(4):e4406.

[18] Ali N, Rahman S, Islam S, Haque, T, Molla NH, Sumon AH, et al. The relationship between serum uric acid and lipid profile in Bangladeshi adults. BMC Cardiovasc Disord, 2019,19(1):42.

[19] Peng TC, Wang CC, Kao TW, Chan JY, Yang YH, Chang YW, et al. Relationship between hyperuricemia and lipid profiles in US adults. Biomed Res Int, 2015,2015:127596.

[20] Levey AS, Stevens LA, Schmid CH, Zhang, YL, Castro AF Rd, Feldman HI, et al. A new equation to estimate glomerular filtration rate. Ann Intern Med, 2009,150(9):604-612.

[21] Zhu JR, Gao RL, Zhao SP, Lu GP, Zhao D, Li JJ, et al. 2016 Chinese guidelines for the management of dyslipidemia in adults. J Geriatr Cardiol, 2018,15(1):1-29.

[22] Bos MJ, Koudstaal PJ, Hofman A,Witteman JC, Breteler MM. Uric acid is a risk factor for myocardial infarction and stroke: the Rotterdam study. Stroke, 2006,37(6):1503-1507.

[23] Holme I, Aastveit AH, Hammar N, Jungner, I, Walldius G. Uric acid and risk of myocardial infarction, stroke and congestive heart failure in 417,734 men and women in the Apolipoprotein MOrtality RISk study (AMORIS).J Intern Med, 2009,266(6):558-570.

[24] Noguchi Y, Tatsuno I, Suyama K,Shibata T, Yoshida T, Otsuka Y, et al. Effect of fenofibrate on uric acid metabolism in Japanese hyperlipidemic patients. J Atheroscler Thromb, 2004,11(6):335-340.

[25] Milionis HJ, Kakafika Al, Tsouli SG, Athyros VG, Bairaktari ET, Seferiadis Kl, et al. Effects of statin treatment on uric acid homeostasis in patients with primary hyperlipidemia. Am Heart J, 2004,148(4):635-640.

[26] Zhou H, Wang Y, Cui L, Chen Y, Li C, Zhao J. The ongoing role of serum uric acid in blood pressure. Clin Exp Hypertens, 2017,39(7):601-605.

[27] Lyngdoh T, Marques-Vidal P, Paccaud F, Preisig M, Waeber G, Bochud M, et al. Elevated serum uric acid is associated with high circulating inflammatory cytokines in the population-based Colaus study. PLoS One, 2011,6(5):e19901.

[28] Patterson R A, Horsley E T, Leake D S. Prooxidant and antioxidant properties of human serum ultrafiltrates toward LDL: important role of uric acid[J]. J Lipid Res, 2003,44(3):512-521.

[29] Konishi M, Iso H, Komachi Y, Shimamoto T, Jacobs DR Jr, Terao A, et al. Associations of serum total cholesterol, different types of stroke, and stenosis distribution of cerebral arteries. The Akita Pathology Study. Stroke, 1993,24(7):954-964.

\section{Tables}




\begin{tabular}{|c|c|c|c|c|c|c|c|c|c|}
\hline & Total & & & Without dyslip & demia & & With dyslipider & & \\
\hline$N$ & Cases $(n=355)$ & Controls $(n=355)$ & $P$ & Cases $(n=97)$ & Controls $(n=113)$ & $P$ & Cases $(n=258)$ & Controls $(n=242)$ & $P$ \\
\hline Age, year & $67.2 \pm 10.2$ & $67.2 \pm 10.2$ & 0.911 & $67.6 \pm 10.0$ & $67.0 \pm 11.4$ & 0.705 & $67.1 \pm 10.2$ & $67.2 \pm 9.6$ & 0.903 \\
\hline Male, n(\%) & $177(49.9 \%)$ & $177(49.9 \%)$ & 1.000 & $45(46.4 \%)$ & $45(39.8 \%)$ & 0.338 & $133(51.6 \%)$ & $133(55.0 \%)$ & 0.445 \\
\hline $\begin{array}{l}\text { Current } \\
\text { smoking, } n(\%)\end{array}$ & $71(20.0 \%)$ & $75(21.1 \%)$ & 0.71 & $16(16.5 \%)$ & $24(21.2 \%)$ & 0.383 & $55(21.3 \%)$ & $51(21.1 \%)$ & 0.947 \\
\hline $\begin{array}{l}\text { Current } \\
\text { dringking, } \mathrm{n}(\%)\end{array}$ & 70 (19.7\%) & $89(25.1 \%)$ & 0.087 & $13(13.4 \%)$ & $30(26.5 \%)$ & 0.019 & $57(22.1 \%)$ & $59(24.4 \%)$ & 0.545 \\
\hline $\mathrm{BMI}, \mathrm{kg} / \mathrm{m} 2$ & $26.2 \pm 4.5$ & $25.6 \pm 3.8$ & 0.058 & $25.2 \pm 5.1$ & $24.5 \pm 3.7$ & 0.250 & $26.6 \pm 4.3$ & $26.1 \pm 3.8$ & 0.201 \\
\hline $\mathrm{SBP}, \mathrm{mmHg}$ & $157.5 \pm 22.0$ & $148.4 \pm 21.2$ & $<0.001$ & $158.9 \pm 26.1$ & $147.0 \pm 20.5$ & $<0.001$ & $156.9 \pm 20.3$ & $149.1 \pm 21.6$ & $<0.00^{\circ}$ \\
\hline DBP, mmHg & $93.8 \pm 16.0$ & $86.6 \pm 12.5$ & $<0.001$ & $94.3 \pm 16.0$ & $87.4 \pm 12.4$ & $<0.001$ & $93.6 \pm 16.1$ & $86.3 \pm 12.5$ & $<0.00^{\circ}$ \\
\hline SUA, mg/dl & $5.8 \pm 1.7$ & $5.5 \pm 1.4$ & 0.006 & $5.2 \pm 1.5$ & $5.5 \pm 1.4$ & 0.208 & $6.0 \pm 1.7$ & $5.5 \pm 1.4$ & $<0.00^{\circ}$ \\
\hline FBG, mmol/L & $6.2 \pm 2.4$ & $6.0 \pm 1.9$ & 0.172 & $5.7 \pm 1.8$ & $5.7 \pm 1.3$ & 0.745 & $6.4 \pm 2.6$ & $6.1 \pm 2.1$ & 0.140 \\
\hline $\mathrm{TC}, \mathrm{mmol} / \mathrm{L}$ & $5.6 \pm 1.2$ & $5.4 \pm 1.2$ & 0.041 & $4.5 \pm 0.5$ & $4.4 \pm 0.5$ & 0.324 & $6.0 \pm 1.1$ & $5.8 \pm 1.2$ & 0.174 \\
\hline $\mathrm{TG}, \mathrm{mmol} / \mathrm{L}$ & $1.5 \pm 0.8$ & $1.4 \pm 0.9$ & 0.349 & $1.0 \pm 0.3$ & $1.0 \pm 0.4$ & 0.328 & $1.7 \pm 0.9$ & $1.6 \pm 1.0$ & 0.771 \\
\hline $\mathrm{HDL}-\mathrm{C}, \mathrm{mmol} / \mathrm{L}$ & $1.6 \pm 0.4$ & $1.6 \pm 0.4$ & 0.205 & $1.6 \pm 0.4$ & $1.5 \pm 0.3$ & 0.238 & $1.7 \pm 0.4$ & $1.6 \pm 0.5$ & 0.483 \\
\hline LDL-C, mmol/L & $3.3 \pm 0.8$ & $3.2 \pm 0.9$ & 0.115 & $2.5 \pm 0.4$ & $2.5 \pm 0.4$ & 0.868 & $3.6 \pm 0.7$ & $3.5 \pm 0.8$ & 0.322 \\
\hline $\begin{array}{l}\text { eGFR, } \\
\mathrm{ml} / \mathrm{min} / 1.73 \mathrm{~m} 2\end{array}$ & $93.9 \pm 16.7$ & $95.8 \pm 14.1$ & 0.100 & $95.1 \pm 16.9$ & $95.7 \pm 14.9$ & 0.814 & $93.4 \pm 16.6$ & $95.8 \pm 13.7$ & 0.074 \\
\hline $\begin{array}{l}\text { Hypertension, } \\
\mathrm{n}(\%)\end{array}$ & $233(65.6 \%)$ & 198 (55.8\%) & 0.007 & $61(62.9 \%)$ & $66(58.4 \%)$ & 0.508 & $172(66.7 \%)$ & $132(54.5 \%)$ & 0.006 \\
\hline Diabetes, n(\%) & $26(7.3 \%)$ & 40 (11.3\%) & 0.070 & $3(3.1 \%)$ & $9(8.0 \%)$ & 0.129 & $23(8.9 \%)$ & $31(12.8 \%)$ & 0.161 \\
\hline $\begin{array}{l}\text { Anti- } \\
\text { hypertensive } \\
\text { drugs, } n(\%)\end{array}$ & $176(49.6 \%)$ & $125(35.2 \%)$ & $<0.001$ & $41(42.3 \%)$ & 36 (31.9\%) & 0.119 & $135(52.3 \%)$ & 89 (36.8\%) & $<0.00^{\circ}$ \\
\hline $\begin{array}{l}\text { Glucose- } \\
\text { lowering drugs, } \\
\mathrm{n}(\%)\end{array}$ & $9(2.5 \%)$ & $18(5.1 \%)$ & 0.077 & $2(2.1 \%)$ & $2(1.8 \%)$ & 0.877 & $7(2.7 \%)$ & $16(6.6 \%)$ & 0.038 \\
\hline
\end{tabular}

Values of continuous variables are presented as mean \pm SD, categorical variables are presented as $n(\%)$.

Abbreviations: BMI body mass index, SUA serum uric acid, SBP systolic blood pressure, DBP diastolic blood pressure, FBG fasting blood glucose, TG triglyceride, TC total cholesterol, HDL-C high density lipoprotein cholesterol, LDL-C low density lipoprotein cholesterol, eGFR estimated glomerular filtration rate. 


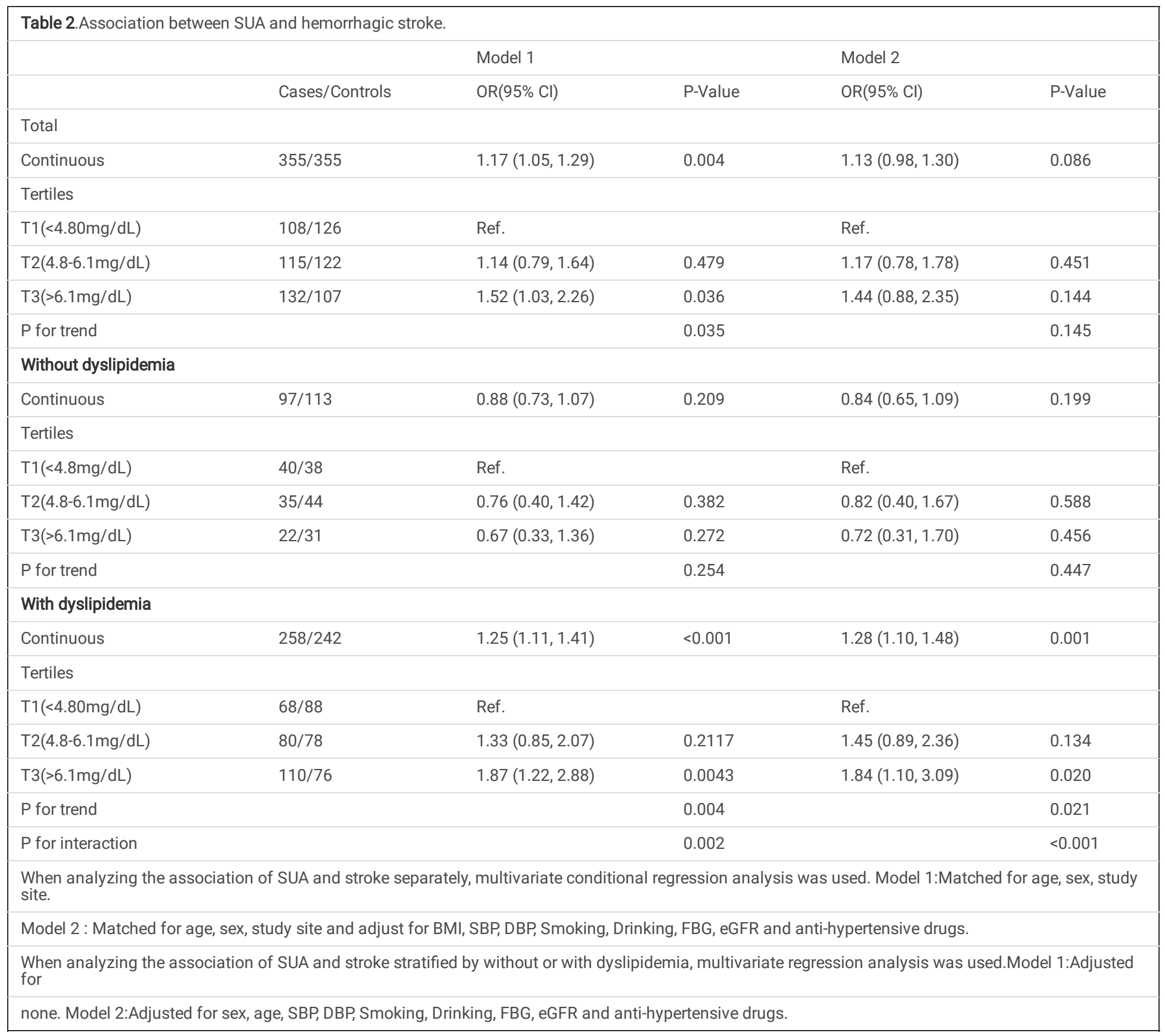

\section{Figures}




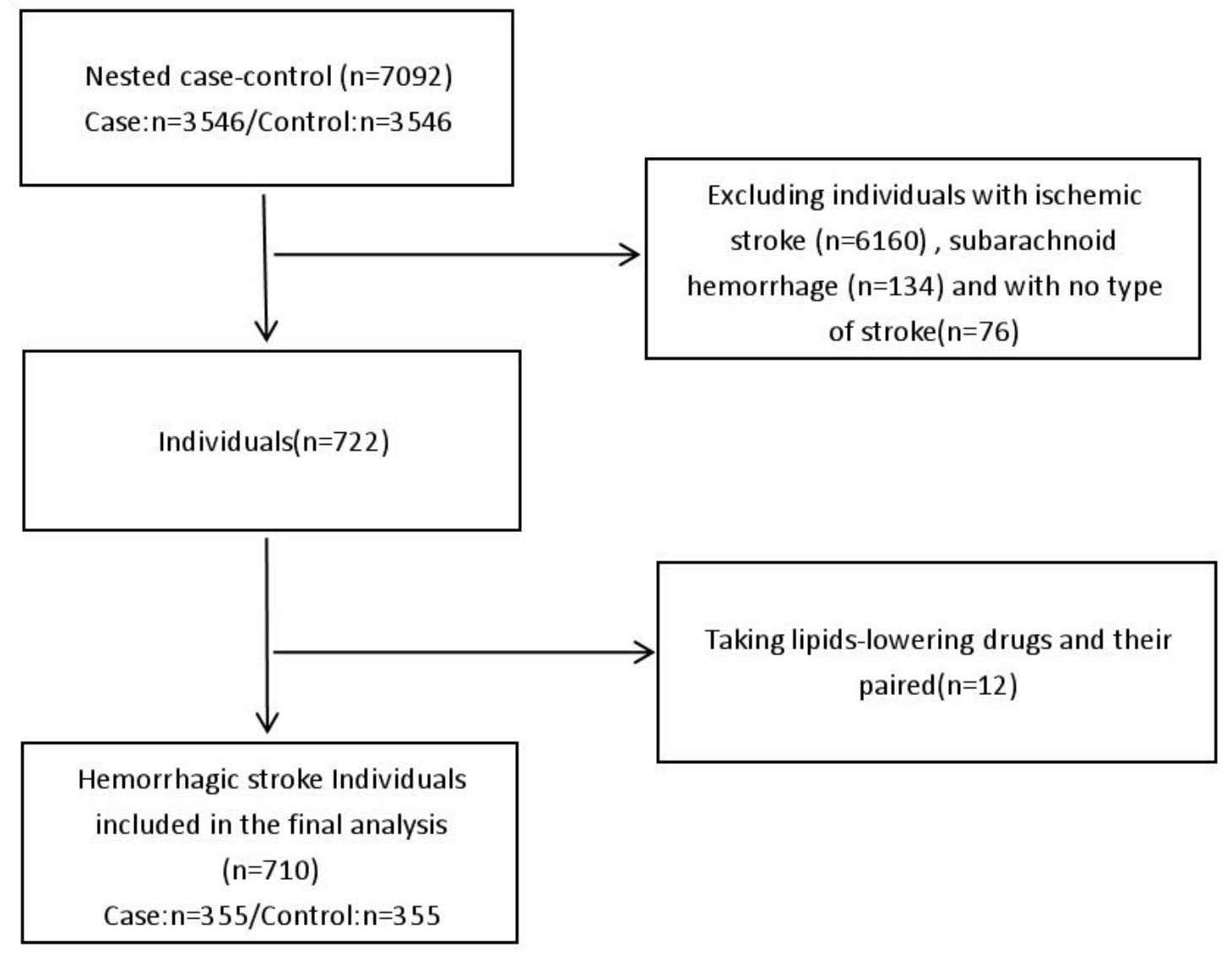

Figure 1

Flow chart of study population. 


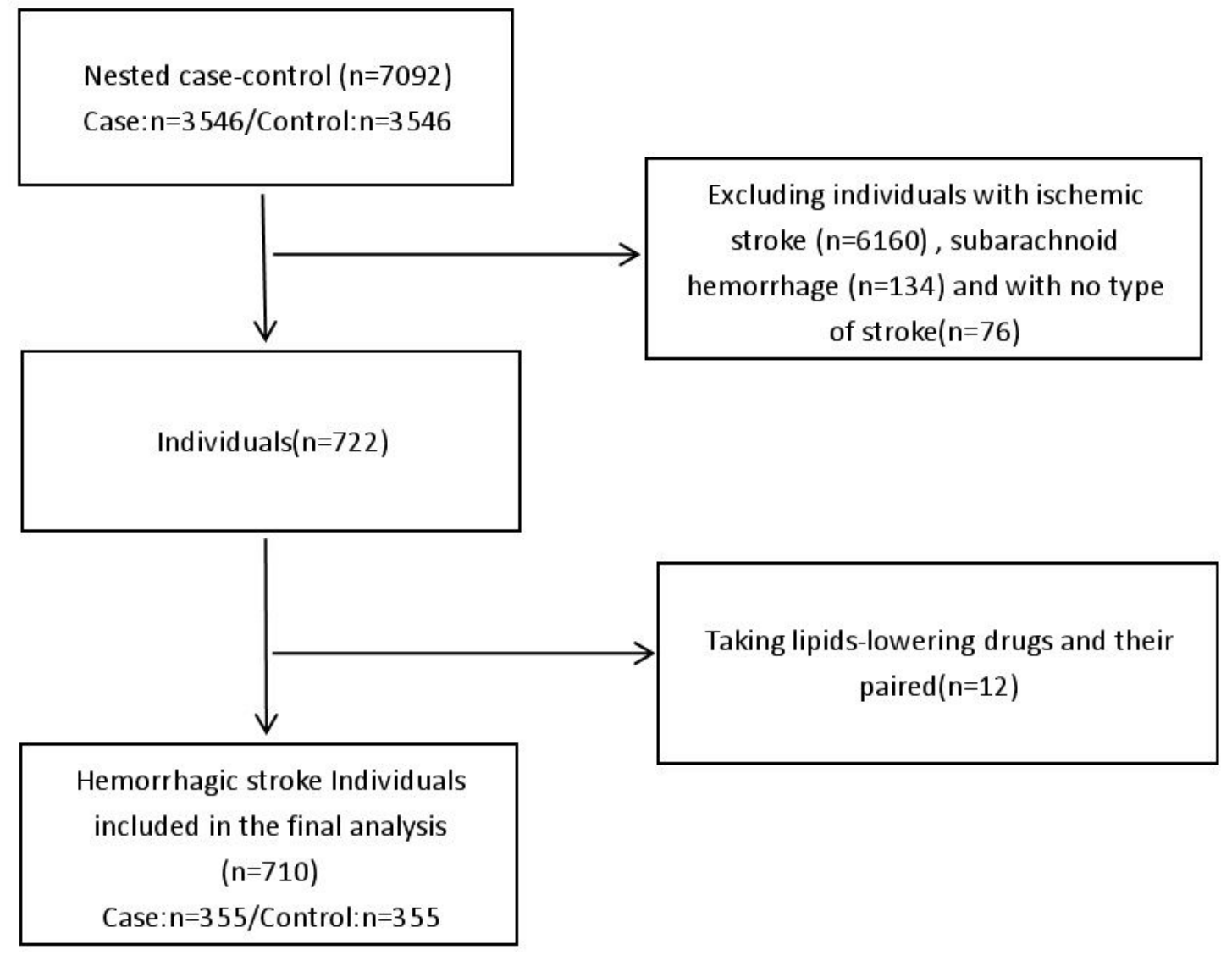

Figure 1

Flow chart of study population.

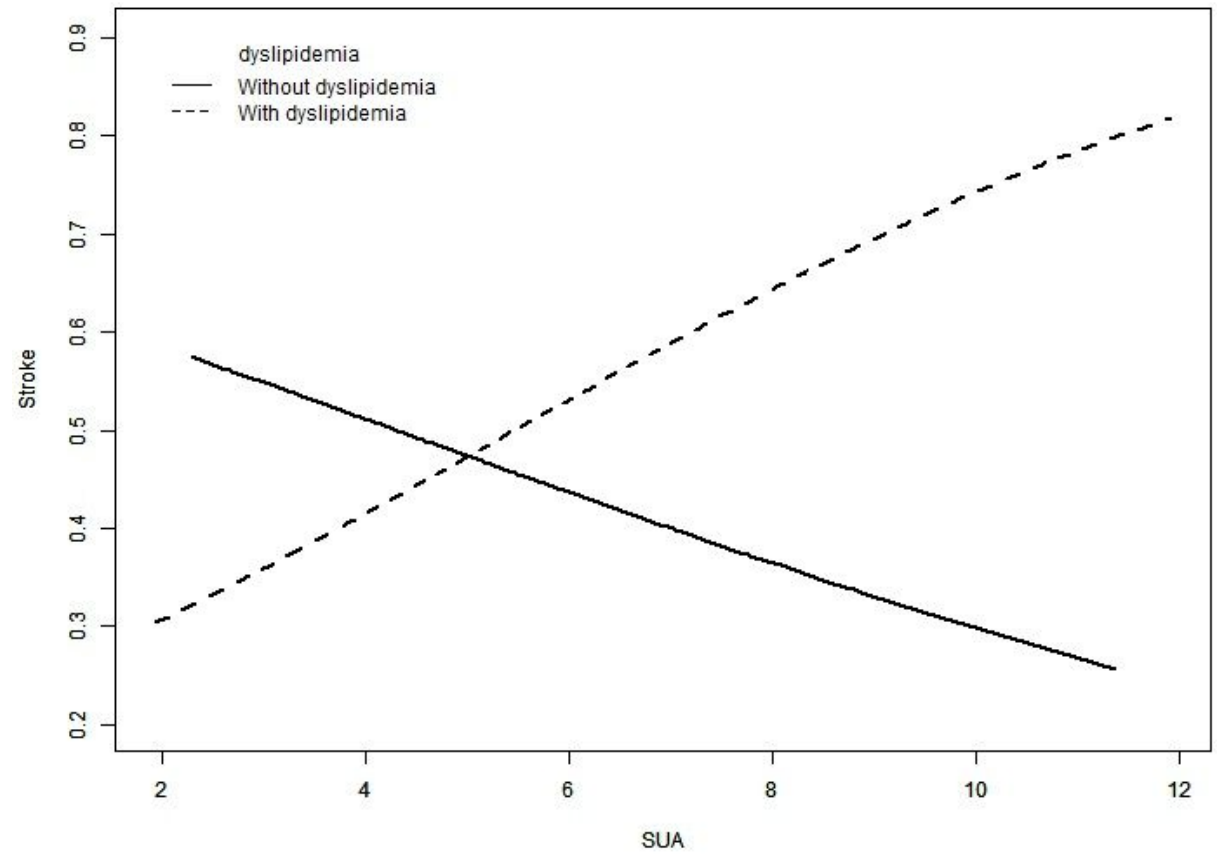

Page 10/13 
Figure 2

The association between SUA and hemorrhagic stroke grouped by with dyslipidemia(dotted line) and Without dyslipidemia (full line). Matched for age, sex, study site and adjust for BMI, SBP, Smoking, Drinking, FBG, eGFR and anti-hypertensive drugs.

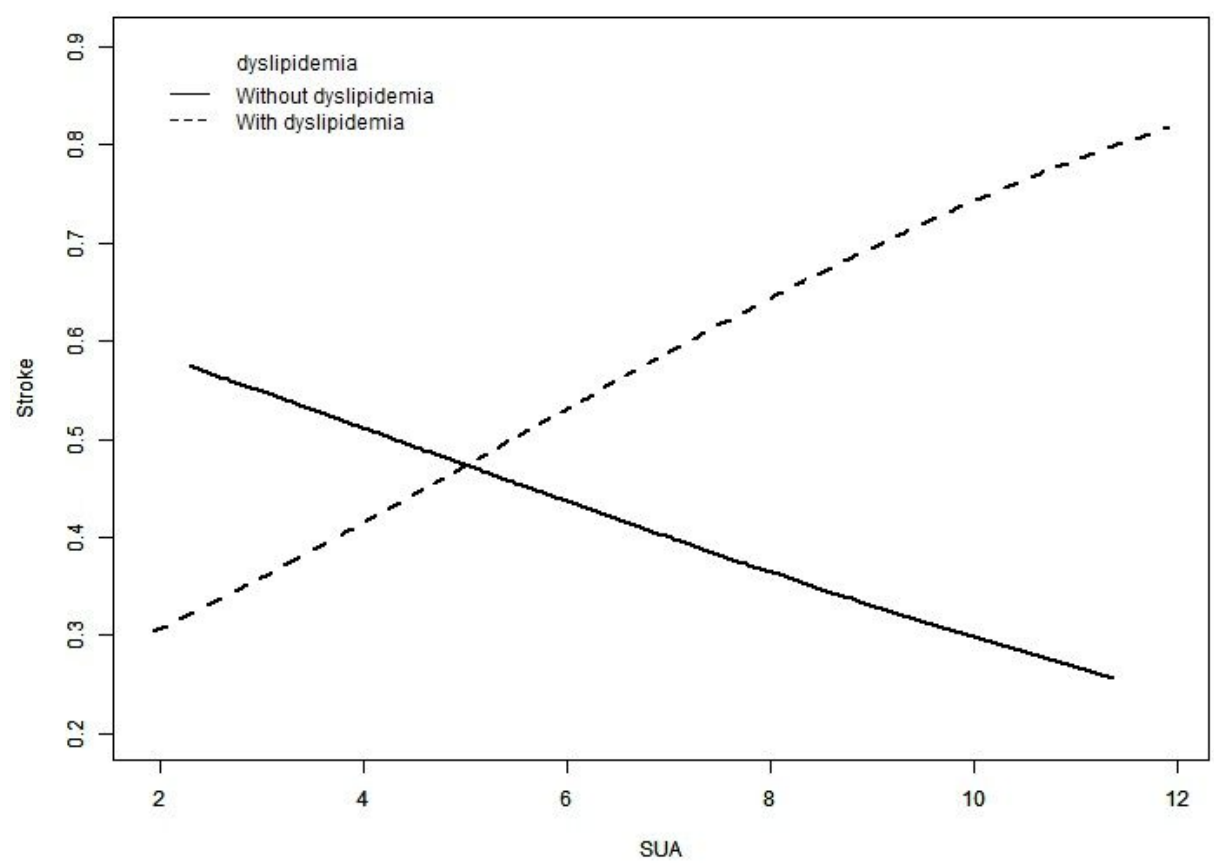

\section{Figure 2}

The association between SUA and hemorrhagic stroke grouped by with dyslipidemia(dotted line) and Without dyslipidemia (full line). Matched for age, sex, study site and adjust for BMI, SBP, Smoking, Drinking, FBG, eGFR and anti-hypertensive drugs. 


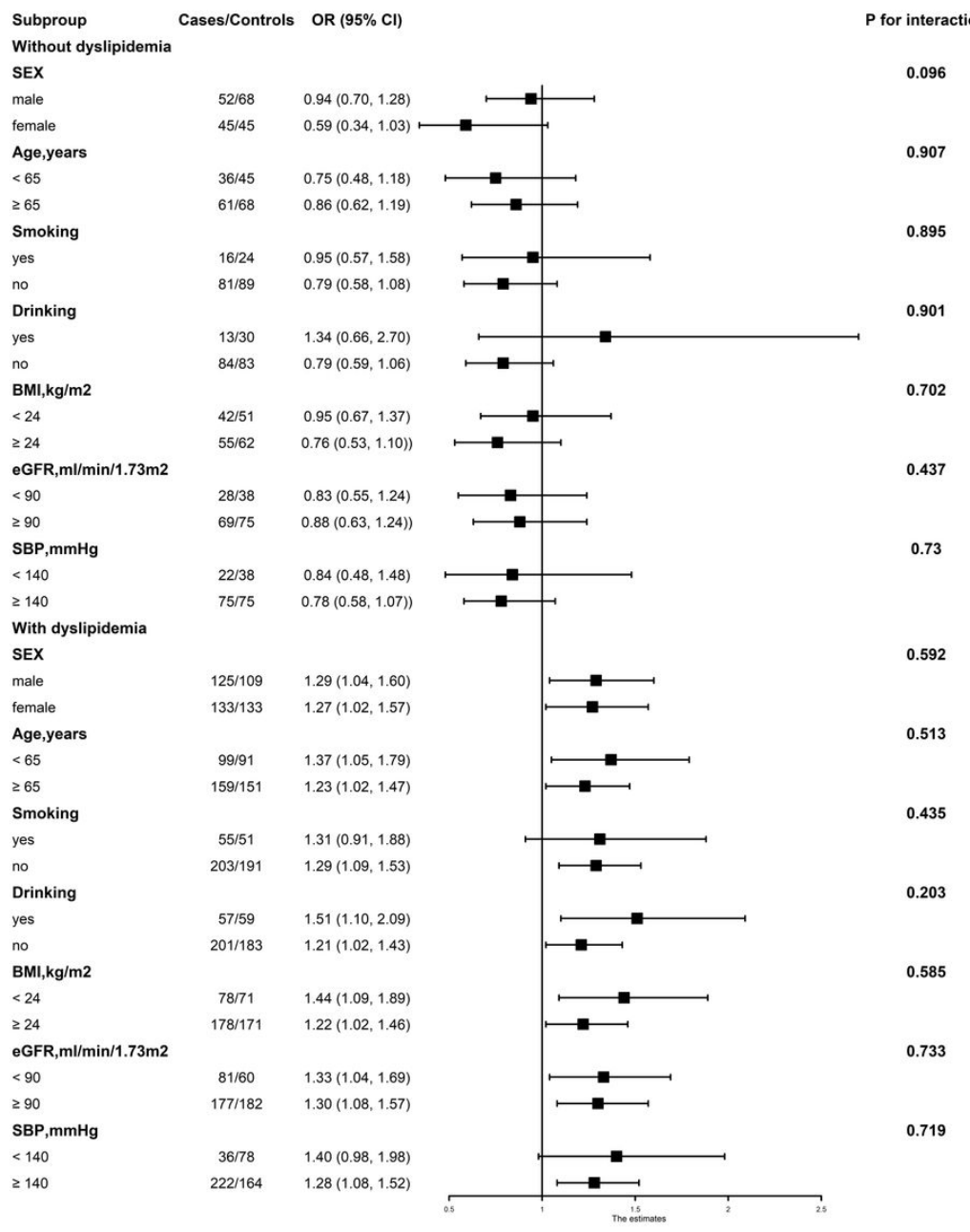

\section{Figure 3}

The association between SUA and hemorrhagic stroke grouped by with dyslipidemia and Without dyslipidemia in subgroup analysis. 


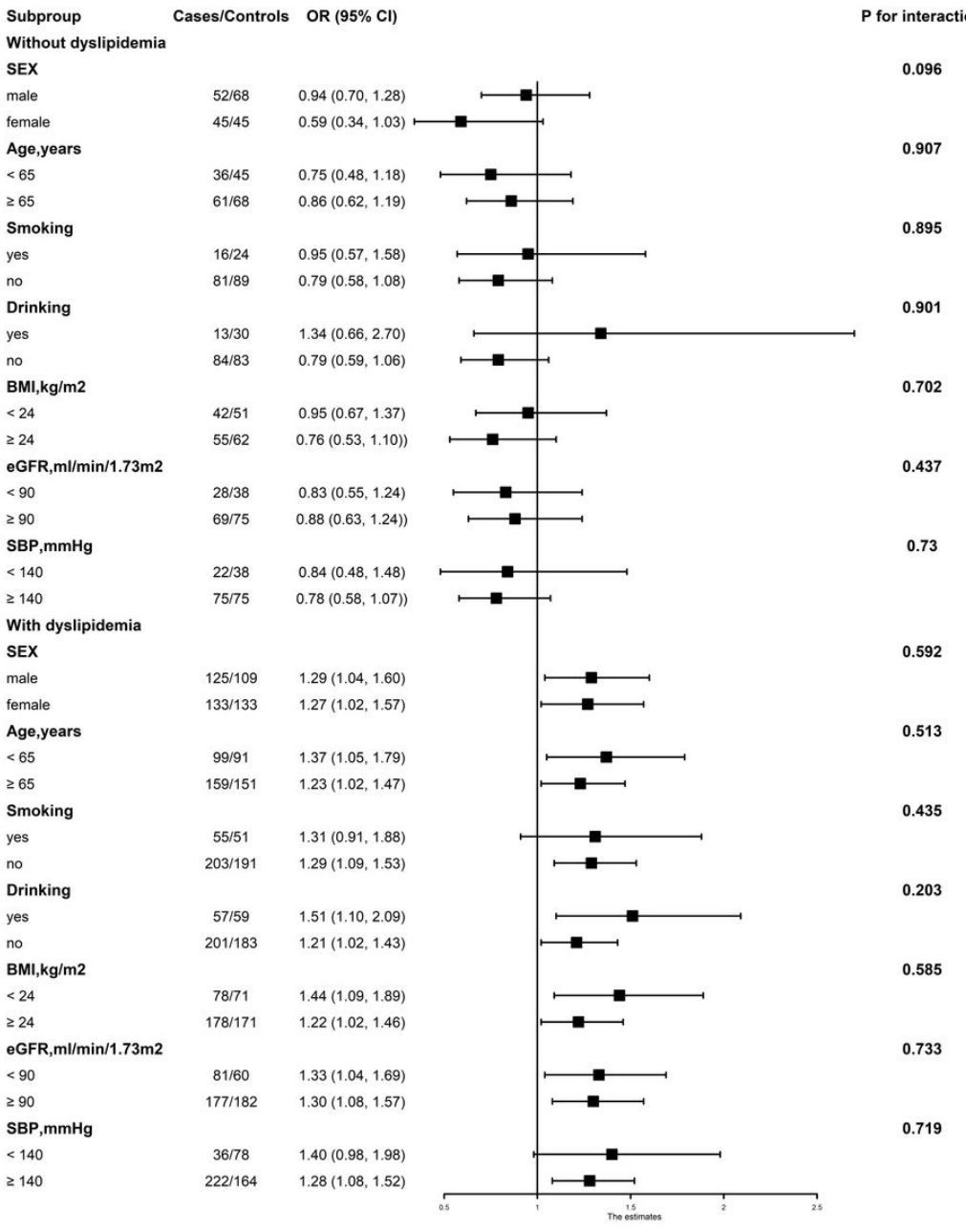

\section{Figure 3}

The association between SUA and hemorrhagic stroke grouped by with dyslipidemia and Without dyslipidemia in subgroup analysis.

\section{Supplementary Files}

This is a list of supplementary files associated with this preprint. Click to download.

- SupplementaryFigure1.png

- SupplementaryFigure1.png

- SupplementaryTable1.docx

- SupplementaryTable1.docx

- SupplementaryTable2.docx

- SupplementaryTable2.docx 